\title{
Caracterización del sector pesquero en el Humedal Nacional Térraba-Sierpe, de noviembre de 2012 a abril de 2013
}

Recibido: 8 de setiembre 2016

Revisado: 10 de octubre 2016

Aprobado: 15 de noviembre 2016

Cindy Arias Bogantes

Costarricense. Máster en

Gestión Integrada de Áreas

Costeras Tropicales por la

Universidad de Costa Rica (UCR), Licenciada en Manejo de Recursos Naturales por la

Universidad Estatal a

Distancia (UNED), Bachiller

en Agronomía con énfasis

en Economía Agrícola de la

Universidad de Costa Rica.

Docente universitaria y

Bióloga Regente.

Correo electrónico:

carias@uned.ac.cr

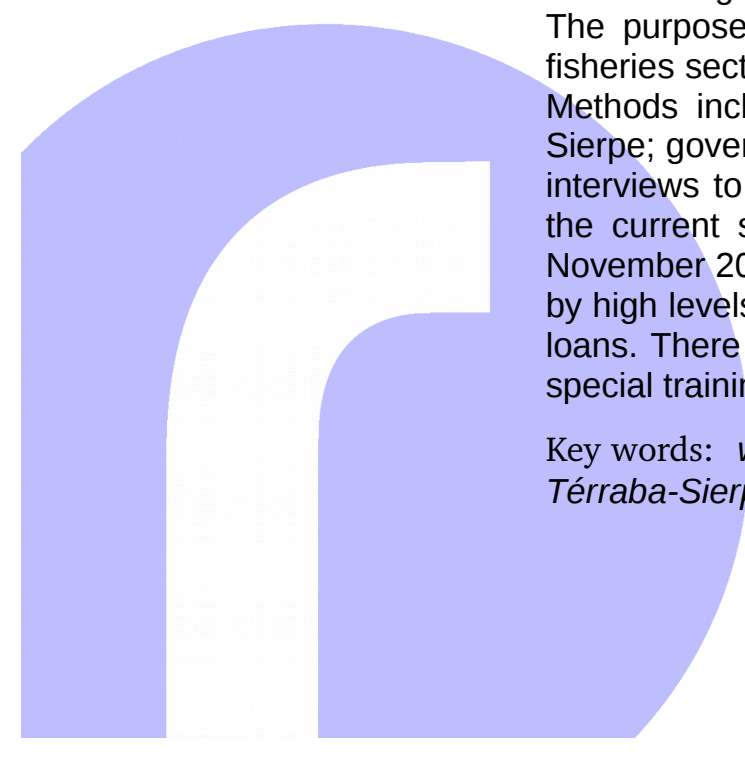

http://investiga.uned.ac.cr/rupturas/

Rev. Rupturas 7(1), Costa Rica, Ene-Jun 2017. ISSN 2215-2466. pp 149-165.
Resumen: El Humedal Nacional Térraba-Sierpe se encuentra ubicado en el cantón de Osa, provincia de Puntarenas (Costa Rica). Dentro de las principales actividades primarias a las que se dedican las comunidades aledañas, se encuentran la pesca y la extracción de moluscos, de las cuales se depende económicamente. El propósito de esta investigación fue desarrollar un análisis del sector pesquero, con el fin de encontrar soluciones que se centraran en una gestión integrada. Los métodos incluyeron visitas a Coronado, Ciudad Cortés y Sierpe, instituciones gubernamentales y recibidores de pescado, así como un total de 75 entrevistas a los pescadores (85\% del total), acerca de su condición socioeconómica y la actividad pesquera. El estudio fue desarrollado entre los meses de noviembre de 2012 y abril de 2013. Socialmente, las comunidades de pescadores se caracterizan por altos niveles de pobreza, bajo nivel de educación y un acceso limitado a los préstamos bancarios. Se presentaron debilidades en cuanto a gestión institucional, educación formal, procesos de capacitación y organización de asociaciones.

Palabras clave: humedal; gestión institucional; actividad pesquera; comunidades pesqueras; Humedal Nacional Térraba-Sierpe.

\section{Characterization of Fishery Sector nearby Térraba-Sierpe National Wetland between November 2012 and April 2013}

Abstract: The Térraba-Sierpe National Wetland is located in Puntarenas province (Costa Rica). Nearby communities are engaged in primary activities such fishing and shellfish extraction, of which the residents are dependent. The purpose of this research was to develop a situational analysis of the fisheries sector, in order to find solutions focused on integrated management. Methods included visits that were made to Coronado, Ciudad Cortés and Sierpe; government institutions, fishing downloads receivers, and a total of 75 interviews to fishers ( $85 \%$ of total about their socio-economic condition, and the current situation of the fishery sector. The study was developed from mber 2012 to April 2013. Socially, fisher communities were characterized

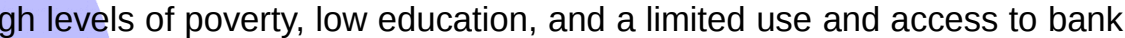
loans. There was a weak in institutional management, formal education, and processes to strengthen associations.

Key words: wetland; institutional management; fisheries; fishing communities; Térraba-Sierpe National Wetland. 
La autora desea expresar un agradecimiento por su colaboración a las siguientes personas:

A la Dra. Daisy Arroyo, al M.Sc. Gerardo Umaña en la Escuela de Biología de la UCR y al M.Sc. Gerardo Cortés de la Escuela de Economía Agrícola y Agronegocios de la misma universidad.

Al Dr. Manuel María Murillo y al M.Sc. Juan Bautista Chavarría, de las escuelas de Biología y Estadística respectivamente, también en la Universidad de Costa Rica.

Al geógrafo Marco Garro por la elaboración de mapas.

A los pescadores y miembros de las asociaciones pesqueras de las comunidades de Coronado de Osa, Ciudad Cortés y Sierpe, así como a los dueños de recibidores de pescado de la zona. Al M.Sc. Jorge Gamboa, coordinador del Programa Nacional de Humedales del Sistema Nacional de Aguas de Áreas de Conservación (SINAC).

\section{Introducción}

Costa Rica posee $51100 \mathrm{~km}^{2}$ de territorio continental y una extensión de aguas marítimas jurisdiccionales de 574725 km² (Comisión Interinstitucional de la Zona Económica Exclusiva de Costa Rica, 2008; Organización de las Naciones Unidas, 2009). Esta zona marítima, poseedora de una gran diversidad biológica, comprende tanto aguas interiores, como las 12 millas de mar territorial, y 188 millas de la zona económica exclusiva; sin embargo, las áreas marinas protegidas solo abarcan un $0,7 \%$ del total (Decimotercer Informe Estado de la Nación 2007).

En su costa Pacífica, con una longitud de 1016 km (y 212 km en la costa Atlántica) (FAO 2011), han sido reportadas un total de 350000 ha. de humedales, de las cuales 30654 ha pertenecen al Humedal Nacional Térraba-Sierpe, que incluye el manglar más grande del país (Simard, Zhang, Rivera, Ross, Ruiz, Castañeda-Moya, Twilley y Rodríguez 2006).

Las actividades económicas dentro de la zona del humedal se han desarrollado productivamente a mediana escala, de forma artesanal y para autoconsumo. Las principales actividades económicas son la extracción de piangua, la pesca y, en años anteriores, la producción de carbón de mangle (esta última ya no se realiza de acuerdo con lo estipulado en la Ley Forestal 7575 de 1995), la acuicultura de camarón (a mediana escala y reiniciada como actividad a partir del 2013), así como la agricultura y ganadería extensiva (Sierra, Castillo y Arguedas 2007).

Por lo anterior, se destaca que el desarrollo socioeconómico de la zona sur del país, y particularmente de la zona del Humedal Nacional Térraba-Sierpe, ha dependido en parte de la actividad pesquera hecha a pequeña escala, por lo que ha sido una de las principales actividades de subsistencia y fuente de alimentos para el consumo familiar. Además, también la pesca ha representado una actividad sociocultural de la zona que se desarrolla de forma complementaria a la extracción de la piangua, la cual se considera la principal ocupación de los pobladores costeros de la zona (Chicas 1995).

En países de América Central y Sur, las condiciones socioeconómicas que caracterizan a los grupos de pescadores artesanales son su bajo nivel económico y educativo. Además, la realización de este oficio se origina por tradición familiar, en que el sector joven de la población se incorpora a la pesca desde temprana edad, y habitan en puertos o comunidades cerca de la costa o de los esteros, desde donde se desplazan hasta las áreas de pesca. Para muchos de ellos la pesca es la actividad que les provee el dinero 
para satisfacer sus necesidades básicas de alimentación, educación, vestido y salud (Beltrán Turriago 2001; Hernández García 2011).

Actualmente, en la zona de influencia del Humedal Nacional Térraba-Sierpe, se encuentran cuatro agrupaciones de pescadores: la Asociación de Empresarios Unidos de Coronado de Osa (Javier Castillo Pescador 2012, comunicación personal), la Asociación de Pescadores Artesanales de Ciudad Cortés y Bocas del río Térraba (ASOPESCAR), la cual se fundó para la protección de la actividad de la pesca y el establecimiento de mecanismos de financiamiento entre los asociados (Irma Picado y Rafael Elizondo Pescadores 2012, comunicación personal); la Asociación de Piangüeros y Recursos Marinos de Ajuntaderas y afines (APREMAA) y la Asociación Mar Nuestro en Dominicalito (IFAM 2010; Registro Nacional de Costa Rica 2013, entrevista a pescadores 2013), las cuales, al igual que las demás, trabajan con el fin de buscar soluciones a los problemas del sector pesquero de la zona, y gestionar ayudas, sociales y económicas de manera integral para sus asociados. En este estudio se consideraron solo las tres primeras, por estar más cercanas y vinculadas con el humedal.

En la investigación se analizó el estado del arte del sector pesquero y se plantean posibles soluciones y respuestas de gestión que esperan favorecer un desarrollo social y económico en armonía con la conservación y uso racional del recurso pesquero. Para ello se evaluaron las organizaciones y los procesos y características del sector, con el fin de plantear orientaciones estratégicas a corto y mediano plazo que permitan una gestión integrada de la actividad pesquera en el Humedal Nacional Térraba-Sierpe.

\section{Materiales y métodos}

Esta investigación se realizó en las comunidades aledañas al Humedal Nacional Térraba-Sierpe (HNTS. Políticamente, esta área incluye a los distritos de Coronado de Osa, Puerto Cortés, Sierpe y Palmar Norte y Sur del cantón de Osa) (Rojas, Alfaro, Solano, Araya y Villalobos 2011).

La zona de influencia general del humedal incluye desde la cuenca del río Grande de Térraba hasta la cordillera Costanera y la cuenca alta del río Sierpe, cuyas extensiones son $4767 \mathrm{~km}^{2}$ y $1960 \mathrm{~km}^{2}$, respectivamente (Bravo, Miranda, Rivera y González 1998). Estas cuencas comprenden los municipios de Pérez Zeledón, Buenos Aires, Coto Brus y Osa (Cedeño, López, Villalobos y Hernández 2012). Los humedales de la cuenca baja de estos ríos desembocan básicamente en seis esteros mayores, que popularmente se identifican como "bocas", entre los que se encuentran: Zacate, Brava, Coronado, Guarumal, Sierpe y Chica (Reyes, Miranda, Monge y Salas 2004).

Para esta investigación, se llevaron a cabo giras de observación quincenal o mensual a la zona del Humedal durante el periodo comprendido entre los meses de noviembre de 2012 y de enero a abril de 2013. Se entrevistó a 
pescadores artesanales de las tres áreas (Coronado, Sierpe y Ciudad Cortés), así como a representantes de instituciones públicas relacionadas con el humedal y la actividad pesquera. Adicionalmente se visitó a los recibidores de pescado de Ciudad Cortés, donde se evaluaron variables pesqueras, socioeconómicas, perspectivas sobre el recurso pesquero en el humedal y la comercialización.

Se procedió a buscar datos estadísticos en registros nacionales del Instituto Nacional de Estadística y Censos (INEC), en la Oficina Regional del Ministerio de Educación Pública y el Ministerio de Salud, relacionados con las comunidades cercanas al Humedal Nacional Térraba-Sierpe. Se analizaron aspectos como actividades económicas, situación poblacional, servicios básicos, nivel educativo formal y condiciones de salud. Lo anterior con el fin de valorar la situación socioeconómica de las comunidades y proponer posibles soluciones dirigidas a la participación de los pobladores dentro de un manejo integrado.

Los grupos meta de estudio fueron los pescadores artesanales aledaños al humedal, así como los encargados de instituciones gubernamentales (ocho instituciones estatales) relacionadas con el sector pesquero (MINAE, Municipalidad de Osa, MAG, Ministerio de Salud, INCOPESCA, Ministerio de Educación circuitos 07, 08, 09 Osa, IMAS y SENASA), y los responsables de los recibidores de pescado. Para efectos de este proyecto, no se incluyó a los pescadores de Dominicalito por dificultades para contactar y reunir a los asociados y porque su actividad directa en el humedal es baja. Se encuestó a 75 pescadores (de un total aproximado de ciento veinte) de las tres comunidades, lo cual tiene un nivel de significancia de la muestra total del 85\% de la probabilidad máxima, según Moya (2009).

Con las encuestas se recopilaron datos relacionados con la cantidad de miembros por familia, condiciones de vivienda, nivel de escolaridad y servicios básicos con los que cuenta la comunidad. Dichas informaciones se analizaron por medio de estadísticas descriptivas, generalidades de los datos como promedios y variación de frecuencias de cada una de las variables consideradas (Canavos 1998).

Se efectuó una determinación de las variables por tema (aspectos socioeconómicos de las comunidades, gestión institucional y pesca y comercialización), y análisis de cada una de ellas por medio de indicadores (se exponen en la descripción de cada una de las secciones), que explicarán la variabilidad y relación entre ellos (Hernández, Fernández y Baptista 2006). Para el análisis de datos económicos y sociales, se elaboraron gráficos mediante la utilización de paquetes estadísticos (Excel Windows 7 y Past software versión $2.7,2012$ ), con el fin de procesar la información obtenida en las encuestas (Canavos 1998) 


\section{Estado de la cuestión}

Según el INEC (2011), la densidad poblacional del cantón de Osa se estima en 29433 habitantes (según último censo poblacional realizado en 2011). De esta cifra, 588 viven en Coronado, 5176 en Ciudad Cortés y 871 en Sierpe; el total de familias en estas comunidades fue de 2327 (Censo de poblaciones, INEC 2011).

Según el Plan de Desarrollo Municipal del Cantón de Osa (2003-2007) (Municipalidad de Osa 2013), el 21,3 \% de la población era pobre y el 36,8\% de los hogares del cantón vivían en la pobreza. A partir de datos obtenidos por el Centro Centroamericano de Población de la Universidad de Costa Rica (2011), se considera a las comunidades de estudio entre las de mayor pobreza en el país. Las causas atribuidas a esta situación han sido la falta de oportunidades de empleo debido a factores como bajas posibilidades de comercio, los bajos niveles de escolaridad de sus habitantes, y la producción de cultivos agrícolas en gran escala, propiedad de grandes compañías. Estas situaciones han obligado a los pobladores de las comunidades a desarrollar actividades para su subsistencia económica, a corto y mediano plazo, mediante la explotación del recurso (pesca y extracción de moluscos) de manera no controlada.

Asociado a ello, la tasa de desempleo en la zona, según datos del INEC (2011), era de un 19,4\%; esta cifra superó el porcentaje nacional en un 3,7\% al reportado para Costa Rica tomando en consideración que las principales fuentes de empleo de la zona de basan en la agricultura, la pesca y la extracción de moluscos.

De acuerdo con lo anterior, una de las principales actividades que se desarrolla en la zona de estudio es la pesca artesanal (18\% entre pesca y extracción de piangua) (INEC 2011). Los resultados del estudio permitieron encontrar que la población de pescadores se distribuye de la siguiente manera: $28 \%$ Coronado, 29\% Sierpe y 43\% Ciudad Cortés (encuestas a pescadores 2013).

De acuerdo con lo anterior, la mayor cantidad de personas dedicadas a la actividad pesquera se encontraba en Ciudad Cortés, específicamente en las comunidades de Ojo de Agua, Renacimiento y la parte céntrica de Ciudad Cortés (esto por ser un lugar más amplio geográficamente y con mayor cantidad de pobladores). En cuanto a la población pesquera de Coronado y Sierpe, se encontraron en igualdad de condiciones con respecto a la cantidad de pescadores; para el primer caso, habitan principalmente las zonas de Punta Mala y Boca Zacate, mientras que en el segundo, Guarumal y Ajuntaderas (Fig. 1) 
Figura 1. Frecuencia de visitación para pesca en el humedal Térraba-Sierpe, de enero a abril 2013

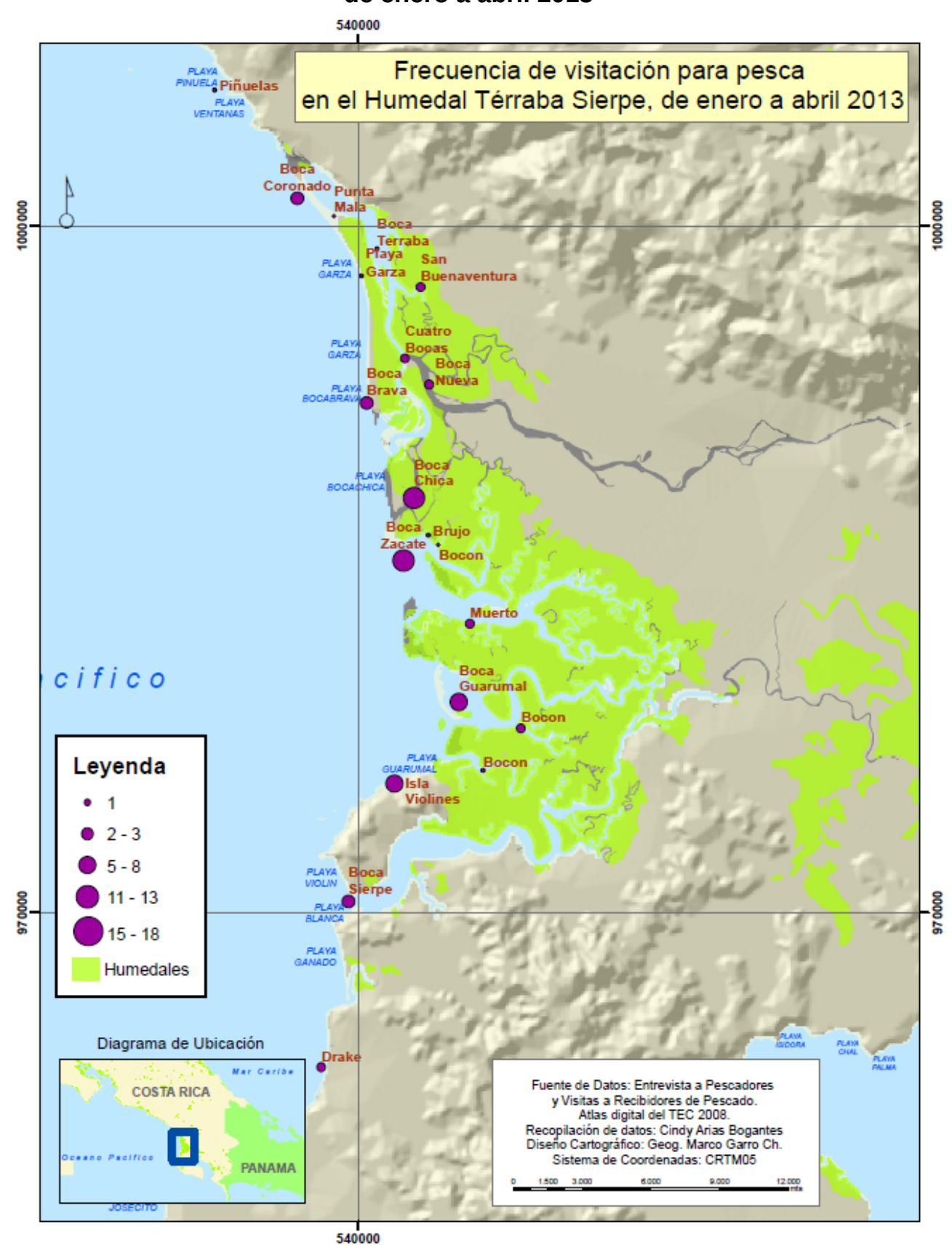

Fuente: elaboración propia.

En relación con la actividad pesquera, según reporte de los pescadores (2013), los ingresos económicos varían dependiendo de la cantidad y tamaño (peso) del producto, las especies capturadas y el comportamiento de los 
precios en el mercado. Se subraya que los recibidores de pescado de la zona son quienes financian el alisto (insumos para cada jornada de pesca) que, en la mayoría de los casos, se rebaja de las ganancias obtenidas. Los ingresos económicos pueden ser semanales o mensuales dependiendo de la duración de cada jornada de trabajo, los cuales van desde menos de 50000 colones a 300000 colones mensuales, en gran cantidad de familias es el único ingreso económico. En caso de que INCOPESCA declare veda (para pesca o piangua), a los pescadores o piangüeros legalmente inscritos y con licencia se les otorga un subsidio de 200000 colones por mes, por familia, a través del IMAS (Entrevista a pescadores 2013; Elida Hernández, IMAS 2013 comunicación personal).

Para el caso del Humedal Nacional Térraba-Sierpe, el producto pesquero se destina a la venta dentro de los mismos recibidores de la zona, por lo que se abastece con él principalmente al mercado interno o de autoconsumo; lo anterior dependerá de la cantidad, el tipo de recurso y del sector donde haya sido pescado. Los ingresos económicos por la obtención y venta del producto, de igual manera, dependen de diferentes condiciones como la época del año (reproducción de las especies, temperatura del agua), de eventos y condiciones ambientales (tormentas, huracanes) (Entrevistas a pescadores 2013). La mayoría de pescadores venden el producto a un recibidor de la zona, y entregan todo lo capturado en la jornada de trabajo sin darle ningún tipo de procesado más que la evisceración. Los recibidores no llevan registros adecuados con información sobre lunas, mareas, lugar y fecha de captura, redes, cantidad vendida por kilo por especie. En algunos casos se tienen registros parciales en las facturas al pagar el producto.

Con respecto al sector educativo en el cantón de Osa, según ACOSA (2008) e INEC (2011), se presenta un rezago en la educación, ya que el porcentaje de instrucción formal es de 89,1\%, encontrándose por debajo de la tasa general del país (92,8\%). Los índices de alfabetización para el país y en particular del cantón de Osa son de 95\% y 91,5\%, respectivamente. Para el año 2001, uno de cada siete niños se encontraban fuera del sistema educativo.

Los más altos índices de analfabetismo se presentan entre los pescadores de avanzada edad y las mujeres, este aspecto influye sobremanera en el desarrollo de la comunicación oral y escrita, la vulnerabilidad socioeconómica con respecto a la posibilidad de opciones de empleo en un mercado laboral estable, interacción, cohesión social y dificultad en la capacidad organizativa (CEPAL 2009). La población joven ha tenido mayor acceso educativo gracias a la creación de más centros educativos y al apoyo de organismos gubernamentales y no gubernamentales dedicados al desarrollo social y a otorgar becas (CEPAL 2009; Adrián Pérez Ministerio de Educación Osa 2013, comunicación personal).

La situación educativa, principalmente de los adultos, obliga a los pobladores de estas comunidades a desarrollar actividades por medio de la explotación del recurso (pesca y extracción de moluscos), de manera no controlada, para su subsistencia económica a corto y mediano plazo. 
En todas las comunidades alrededor del humedal hay centro de enseñanza primaria, excepto en Boca Zacate y Sábalo por encontrarse dentro del humedal, y centros de enseñanza secundaria solamente Palmar, Ciudad Cortés y Sierpe, es decir, en los tres centros de población. Actualmente, el Ministerio de Educación Pública trabaja con el proyecto de alfabetización de adultos y bachillerato por madurez, en particular en las comunidades de piangüeros y pescadores. A pesar de estas oportunidades, se presentan dificultades para llevar a cabo con éxito los programas educativos, pues hay déficit de docentes, problemas con vías de acceso a los centros de enseñanza, alta deserción escolar, falta de recursos económicos por parte de la población y falta de interés por un sistema educativo formal.

En el tema de salud, en las comunidades céntricas de Coronado de Osa, Ciudad Cortés y Sierpe, se ofrece el servicio de Ebais (Atención Primaria en Salud); además, se cuenta con el Hospital de Osa, ubicado en Ciudad Cortés. Adicionalmente, hay Centros de Salud en cada una de las comunidades, excepto en Punta Mala, Boca Zacate, Ajuntaderas, Guarumal y Potrero.

La tasa de natalidad es de un 93,4\%. Esta es más alta que el promedio nacional $(91,7 \%)$ y hay una gran mayoría (30\%) de madres solteras entre los 15 y los 19 años de edad (INEC, 2011). Dentro de las enfermedades más frecuentes entre los pobladores de la zona están: problemas cardiovasculares, problemas respiratorios, tumores, tuberculosis e intoxicaciones (principalmente en relación con el consumo de moluscos en época de marea roja). Además, el $4 \%$ de los niños atendidos en Centros de Educación y Nutrición y Centros Infantiles de Atención Integral (CEN-CINAI) presentan algún grado de desnutrición. Estos servicios se encuentran en los centros de población, Ciudad Cortés, Palmar Norte y Sierpe.

A continuación, se señalan los factores de riesgo presentes en la zona que aumentan la posibilidad de adquirir las enfermedades mencionadas: calidad del agua no apta para el consumo humano (se trabaja con ASADAS en centros de población, las comunidades dentro del humedal no tienen agua potable), mal manejo de desechos sólidos, embarazo adolescente y violencia familiar como patologías sociales. También se anotan la ausencia de rellenos sanitarios, condiciones inadecuadas o precarias de vivienda, mal manejo de sistema de alcantarillado. Por lo tanto se considera al Cantón de Osa, en general, como una de las zonas prioritarias de atención de salud en el país (Ministerio de Salud 2005).

Para el caso de las comunidades pesqueras en la zona de estudio, estas reciben visitas periódicas (diarias, semanales, quincenales o mensuales) por parte de los Asistentes Técnicos de Atención Primaria (ATAP), de los Ebais, dependiendo del riesgo en las condiciones de salud de cada familia.

Con respecto a los servicios básicos, en el cantón de Osa, en general, se cuenta con telefonía (residencial y móvil), abastecimiento de agua potable suministrado principalmente a través de acueductos rurales, electricidad, centros educativos con predominio de escuelas unidocentes y secundarias. 
En materia de salud, se dispone de 18 Ebais y un centro hospitalario. Asimismo, se cuenta con una serie de instituciones estatales (Bancos Estatales, IMAS, MINAE, MAG, MEP, Ministerio de Salud, SENASA), localizadas mayormente en los centros de población (La Gaceta 2012).

Todas las comunidades del sitio de estudio cuentan con servicio de electricidad, a excepción de Potrero, Guarumal, Ajuntaderas y en las bocas, por encontrarse dentro del Humedal, lo cual dificulta llevar el servicio a la zona.

Solamente el centro de Ciudad Cortés, Renacimiento y Ojo de Agua tienen el servicio de agua potable por medio de Acueductos y Alcantarillados, mientras que las comunidades de Palmar Sur, Coronado Centro, Punta Mala, Sierpe, Sábalo, Potrero y Las Fincas obtienen el recurso por medio de la administración de ASADAS; Zacate, Ajuntaderas, Potrero y Guarumal, por medio de pozos que los mismos pobladores han construido.

Los servicios adicionales como teléfono residencial, televisión por cable e internet, solo se encuentran disponibles en centros de población y en familias de clase media o media-alta.

Según las bases de datos de INCOPESCA, Departamento de Protección, Reporte por Base de Operación (INCOPESCA Oficina Regional de Golfito 2013, comunicación personal), y como se comentó anteriormente, para la zona de estudio, hay registradas 45 licencias de pesca activas (legalmente inscritos), con su respectiva embarcación y un total de 190 licencias para extracción de moluscos, a pesar de que en realidad existen, aproximadamente, alrededor de 200 pescadores dentro del humedal (el 90\% realizan la actividad informalmente).

La pesca se considera una de las principales actividades económicas en el humedal. Dentro de los conflictos fundamentales que se tienen al respecto están: el irrespeto de las zonas de pesca (pesca desarrollada dentro del humedal), licencias (alrededor del $85 \%$ no cuenta con los permisos requeridos), la falta de programas de gestión y coordinación entre instituciones y ausencia de su personal técnico y operativo. Según las entrevistas realizadas a los pescadores (2013), entre los aspectos críticos del sector en la zona de estudio se encuentran: la restricción en cuanto al uso de artes de pesca. Para los pescadores es un obstáculo porque solo se visualiza en términos económicos y no desde un punto de vista de la sostenibilidad del recurso; otro de los aspectos es la competencia con barcos camaroneros, y el descontrol en cuanto a la actividad agrícola, la contaminación de las aguas de los ríos Térraba y Sierpe, lo que afecta la cantidad y distribución de los peces, todo esto ligado a la falta de gestión y manejo de forma integrada del humedal.

Según Chicas (1995) y Solís, Madrigal, Barguil y Cambronero (2009), dentro de los principales retos de la actividad pesquera en la zona de estudio se han encontrado los siguientes: escasez del recurso pesquero dentro del manglar, restricción en el otorgamiento y renovación de licencias para pesca o extracción de piangua, ilegalidad en el uso de las artes de pesca, 
competencia con los barcos camaroneros por la disponibilidad del recurso, presión de las autoridades estatales para el funcionamiento de los recibidores y centros de acopio, los bajos precios de mercado, el acceso limitado de las autoridades estatales a los sitios de pesca, las prácticas poco sostenibles de pesca, el turismo no controlado con respecto a uso y capacidad de carga, así como las condiciones desiguales entre la pesca artesanal, la industrial y la semiindustrial.

\section{Resultados}

Según las 75 entrevistas hechas a los pescadores (2013), en relación con el tiempo de vivir en la zona (Coronado, Ciudad Cortés o Sierpe), la gran mayoría (83\%) dice tener menos de diez años de residir en el sitio de estudio, ya que muchos provienen de otros lugares del país o de zonas cercanas o dentro del humedal. Algunas de las familias pescadoras han sido reubicadas en proyectos de vivienda del IMAS, en zonas más céntricas (por ejemplo, ciudadela Renacimiento, Ciudad Cortés), donde se les puede suplir de servicios básicos con el fin de mejorar las condiciones de vida de los pobladores. De estas familias, $77 \%$ cuentan entre uno a cinco miembros y un $23 \%$, de seis a diez personas (que habitan en una casa de proyecto de vivienda); con un promedio general de cuatro integrantes por casa de habitación.

Respecto a las condiciones de vivienda, la mayoría de los pescadores posee casa propia (59\%) (tomando en consideración que estas viviendas las otorgó el IMAS), seguido por un $24 \%$ cuya casa es prestada (por algún familiar o amigo) y una minoría (18\%) que la alquila (por montos entre 20000 a 100 000 colones mensuales). La mayoría de las casas son de bienestar social, construidas con material prefabricado, cemento y, en algunos casos, madera; la disponibilidad de servicios básicos (agua, electricidad, telefonía residencial, transporte público, servicios municipales y adicionales) depende de la posición geográfica, extensión territorial y cantidad de población.

Como se muestra en el Gráfico 1, la mayor cantidad de pescadores se encuentra en Ciudad Cortés, distribuidos en las comunidades de Ojo de Agua, Renacimiento y la parte céntrica, esto por ser un lugar más amplio geográficamente y con mayor cantidad de pobladores que los demás sitios. Coronado y Sierpe se encuentran en igualdad de condiciones a nivel demográfico, la primera con más parte terrestre y la segunda con parte de sus comunidades dentro y fuera del humedal.

Al analizar las actividades económicas realizadas por los grupos de pescadores de la zona, se observó que solo un $16 \%$ se dedica a la pesca (considerada solo como extracción de peces) como única fuente de ingresos económicos. Adicionalmente, fue evidente que la mayoría se dedica a otras actividades adicionales a la pesca como la recolección de piangua con un $64 \%$ (por lo tanto, $80 \%$ de la población está vinculada con la pesca artesanal en la zona), seguida de otras ocupaciones menores como el comercio 
(trabajos temporales en establecimientos de la zona), la construcción (6\%), la jardinería y la agricultura ( $4 \%$ cada una).

\section{Gráfico 1. Distribución de pescadores en la zona de influencia del humedal;} con base en las encuestas a pescadores 2013 y el INEC, 2011.

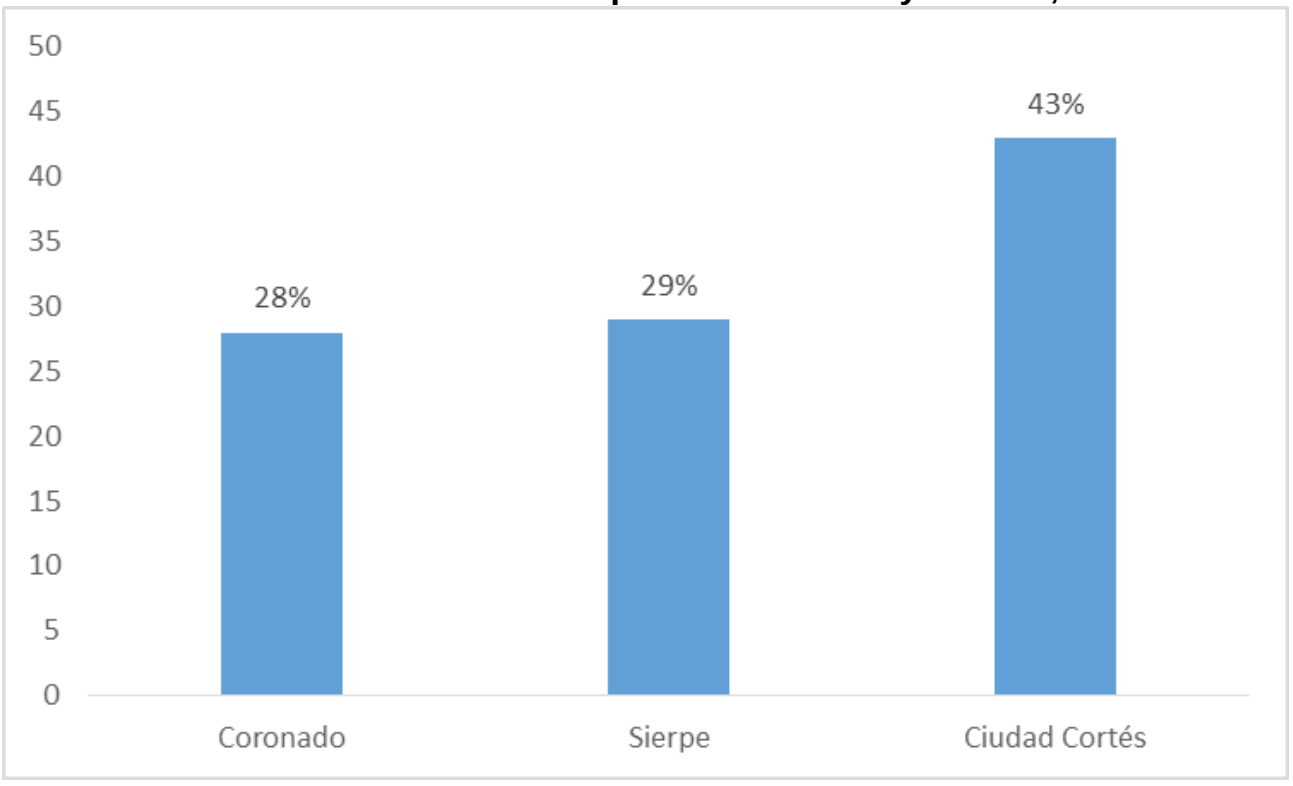

Fuente: Elaboración propia a partir de INEC (2011) y entrevistas realizadas en el 2013.

De acuerdo con datos suministrados por los pescadores, la mayoría (38\%) tiene ganancias mensuales (por pesca o extracción de piangua) entre 50000 y 100000 (un solo salario por familia, ver dato anterior de integrantes por familia). En algunos casos (22\%), hay 2 personas por hogar que laboran en la actividad pesquera (de los cuales generalmente solo hay un permiso o licencia por familia). Los ingresos económicos dependen de muchos factores como la época del año, precios del producto en el mercado, tamaño y especies de peces que se logren obtener.

En lo que respecta al nivel educativo de los pescadores entrevistados, el $46 \%$ no ha completado la primaria (principalmente en zonas alejadas dentro del humedal), seguido de un $38 \%$ con primaria completa y solamente un $15 \%$ ha logrado llegar a la secundaria, pero sin completarla (muchos dejan la educación formal desde niños o adolescentes por la situación económica familiar y se dedican a la actividad pesquera para colaborar con los ingresos en el hogar). Este aspecto se considera una variable en el desarrollo de la comunicación oral y escrita, de la situación socioeconómica en relación con la posibilidad de opciones de empleo y estabilidad laboral, así como un influyente en la capacidad organizativa en el tema de las asociaciones.

De acuerdo con las entrevistas a pescadores, la mayoría se dedica a otras actividades adicionales a la pesca, la extracción de piangua es la principal, 
seguida de comercio, construcción, agricultura y jardinería, todas estas ocupaciones no formales y temporales dependiendo del comportamiento de la actividad pesquera.

A nivel organizacional, la pesca artesanal en el Humedal Nacional TérrabaSierpe es efectuada por grupos de pescadores o piangüeros que se encuentran en la zona de Dominicalito, Coronado de Osa, Ojochal, Ciudad Cortés, Palmar Sur y Sierpe, los cuales se agrupan en asociaciones que se encuentran en las comunidades de Dominicalito (no se contabilizó), Coronado de Osa, Ojochal, Ciudad Cortés, Palmar Sur y Sierpe, pero no cuentan con un centro de acopio para el recibimiento y procesamiento del pescado, por lo que entregan el producto directamente en los recibidores, los cuales, a su vez, sirven como intermediarios de venta, ponen el precio de compra y venta del producto y también fungen como intermediarios a otros establecimientos y ventas comerciales.

De acuerdo con las entrevistas realizadas (2013), la mayor parte de los pescadores (96\%) solo cuentan con una embarcación, con medidas desde los $3 \mathrm{~m}$ hasta los $8 \mathrm{~m}$ de largo, con uno o dos motores que van desde los 3,5 a los 40 caballos de fuerza, un detalle importante es que la mayoría lleva hielera portátil a sus jornadas de pesca (las embarcaciones no las incluyen) de las cuales depende el tiempo laborado y el almacenamiento del producto.

Sobre las artes de pesca, a pesar de que en la Ley de Pesca y el Plan de Manejo del Humedal se prohíbe el uso de trasmallo y líneas por cantidad de anzuelos, en la actualidad, la gran mayoría de los pescadores utilizan cuerda, línea con una variedad de anzuelos variable entre 1000 y 15000 y el trasmallo con tamaños de los 8 a los 60 metros (dependiendo de las especies de interés), ya que, según el criterio de los pescadores, a mayor cantidad de producto, mayores ingresos económicos.

Con respecto a la gestión institucional, durante las entrevistas efectuadas a los actores e instituciones gubernamentales involucradas, se identificaron una serie de problemáticas relacionadas con el sector pesquero, las cuales dificultan el proceso de gestión y manejo integrado del sector en el Humedal Nacional Térraba-Sierpe, dentro de las que se encuentran el incumplimiento en cuanto a zonas de pesca, licencias (alrededor del 85\% no cuenta con los permisos requeridos), artes utilizadas, falta de programas de gestión y coordinación entre instituciones, así como la ausencia de personal técnico y operativo de estas.

Según actores institucionales y pescadores, los aspectos más críticos del sector pesquero en la zona de estudio son las restricciones en cuanto al uso de artes de pesca, lo cual es visualizado por los pescadores solo en términos económicos y no desde el punto de vista de sostenibilidad del recurso, la competencia con barcos camaroneros, el descontrol en cuanto a la actividad agrícola y la contaminación de las aguas de los ríos Térraba-Sierpe, lo que afecta la distribución de los recursos, todo esto ligado a la falta de gestión y manejo de forma integrada. 


\section{Conclusiones}

Los pescadores artesanales de Térraba-Sierpe se localizan en Ciudad Cortés (43\%), en segundo y tercer lugar, Coronado de Osa y Sierpe, esto debido a la extensión de cada uno de los lugares y disponibilidad de servicios básicos.

La gran mayoría de los pescadores se dedica prioritariamente a la extracción de piangua (64\%), en particular en las comunidades de Ciudad Cortés y Sierpe, teniendo como segunda actividad la pesca (peces) artesanal; ambas se consideran la base de la economía de la región junto con la ganadería extensiva, producción de camarón, caña de azúcar, palmito y frutas.

Gran parte de los pescadores pertenece a alguna asociación, dentro de las cuales se presentan conflictos a nivel organizativo, económico y legal, lo que impide un verdadero rendimiento y cumplimiento de los objetivos de la actividad.

En la mayoría de las poblaciones, se cuenta con servicios básicos (electricidad, agua potable, telefonía, servicios de salud y transporte público), con excepción de las comunidades de Guarumal, Ajuntaderas, Punta Mala y Boca Zacate, por encontrarse dentro del humedal y con acceso solo por vía acuática.

A pesar de la existencia de atención médica en el Hospital Central de Osa y Centros de Atención Primaria (EBAIS) en las comunidades, se presentan situaciones que afectan a los pobladores como lo son: falta de agua potable, mal manejo de desechos sólidos, condiciones inadecuadas de vivienda y mal sistema de alcantarillados, por lo que se trabaja en programas de promoción de salud y de prevención de la enfermedad.

El nivel educativo de los pescadores es bajo, con escolaridad de primaria (completa e incompleta), un alto porcentaje de deserción escolar por razones económicas, geográficas y de labores de pesca y piangua como prioridad para obtener ganancias inmediatas. La mayoría de personas se inician en la actividad pesquera desde niños o jóvenes, por lo que abandonan el sistema educativo formal para ayudar económicamente a la familia.

El grado de desarrollo, educación y progreso personal de los pescadores incide en la dinámica del sistema de organización de las asociaciones y del trabajo en equipo de forma conjunta y dinámica.

En las zonas aledañas al humedal se ha dado un crecimiento y expansión agroindustrial, principalmente, de palma aceitera y de arroz, lo cual ha producido una invasión dentro de los límites del humedal, así como un impacto en el uso de suelo y la vulnerabilidad al medio acuático y de la biodiversidad presente en general.

Debido a las situaciones de salud, educación y falta de oportunidades de empleo, la zona de estudio se considera una de las más pobres en el país, lo que obliga a los pescadores a desarrollar actividades de subsistencia.

Entre algunos de los agentes que producen amenazas a las comunidades y al sector pesquero, se encuentran: la contaminación, falta de rellenos 
sanitarios, expansión de frontera agrícola, cambio de uso de suelo, sobreexplotación del recurso pesquero, ilegalización de la actividad dentro del humedal y conflictos sociales en general.

\section{Recomendaciones}

Continuar con los programas de alfabetización de adultos, así como fomentar los programas de motivación dirigidos a niños y jóvenes en etapa escolar, con el fin de disminuir el porcentaje de deserción e incrementar el nivel educativo en la población.

Se recomienda establecer programas o proyectos dirigidos a los pescadores referentes a temas financieros, como el ahorro, de tal manera que logren aprovechar las utilidades y puedan mejorar las condiciones socioeconómicas de sus familias.

Efectuar capacitaciones periódicas dirigidas a los pescadores y piangüeros sobre posibles alternativas de fuentes de empleo o alternativas productivas, y así sea posible fortalecer un sistema de independencia económica y autosuficiencia por medio de proyectos innovadores, los que permitan una solvencia económica y una capacidad emprendedora que satisfaga las necesidades actuales de la población.

Incentivar la creación y la puesta en práctica de programas de salud dirigidos a las comunidades de los pescadores sobre procesos de mejoramiento ambiental, promoción y revisión de la salud de los pobladores y proyectos de deportes y culturales.

El Humedal Nacional Térraba-Sierpe está compuesto por sistemas complejos, a nivel geográfico, histórico y arqueológico, con influencia en los ecosistemas como humedales y manglares, por lo que se deben tomar en cuenta decisiones dentro de un sistema integral y complejo.

Se considera pertinente, dentro del manejo integrado, un enfoque ecosistémico de la actividad pesquera dentro del humedal, de tal manera que la actividad se lleve a cabo de manera sostenible, tomando en cuenta la situación ambiental, social y económica de las comunidades pesqueras.

\section{Bibliografía}

Área de Conservación Osa (ACOSA), Nature Conservancy, Universidad para la Conservación Internacional y Escuela Latinoamericana de Áreas Protegidas. 2008. Proyecto para la elaboración de los planes de manejo de las Áreas Protegidas de ACOSA. Resumen ejecutivo del 
Plan de Manejo del Humedal Nacional Térraba-Sierpe (versión para oficialización). Costa Rica.

Asamblea Legislativa de Costa Rica. 1995. Ley Forestal 7575. Costa Rica.

Beltrán, Claudia. 2001. Aspectos socioeconómicos y técnicos de la pesca artesanal en El Salvador, Costa Rica, Panamá, Ecuador y Colombia. FAO Circular de Pesca N95712. Italia.

Bravo Ramos, Julián, Carlos Miranda Hernández, Luis Rojas Rivera y Jaime González Acosta. 1998. Caracterización de la vegetación de los humedales en la Región Sierpe-Térraba, Osa, Puntarenas. Costa Rica. Ministerio de Obras Públicas y Transportes. Instituto Geográfico Nacional. Informe Semestral 34. Costa Rica.

Cedeño, B., A. López, E. Villalobos y A. Hernández. 2012. Ejes modificadores de las Condiciones Biofísicas de la Cuenca del Río Grande de Térraba. Revista Geográfica de América Central (48). Costa Rica.

Centro Centroamericano de Población. 2011. Proyecciones Distritales de la Población de Costa Rica. Universidad de Costa Rica.

CEPAL. 2009. Impacto Social y Económico del Analfabetismo. Oficina Regional de Educación para Latinoamérica y el Caribe. UNESCO. Chile.

Chicas, Francisco. 1995. Distribución, Diversidad y Dinámica poblacional de la Ictiofauna Comercial de la Reserva Forestal Térraba-Sierpe. Puntarenas, Costa Rica.

Comisión Interinstitucional de la Zona Económica Exclusiva en Costa Rica. 2008. Estrategia nacional para la gestión integrada de recursos marinos y costeros de Costa Rica. INCOPESCA, SINAC, Servicio Nacional de Guardacostas, ICT, MOPT, UCR, UNA, Federación Nacional del Sector Pesquero, MarViva, TNC, Conservación Internacional, CMC-CR. Costa Rica. 
Canavos, George. 1998. Probabilidad y Estadística. Aplicaciones y métodos. Editorial McGraw-Hill: México.

Estado de la Nación. 2007. Decimotercer Informe Estado de la Nación. La Gaceta. Diario Oficial. Costa Rica

Hernández, Milton. 2011. Pescadores en América Latina y el Caribe: espacio, población, producción y política. Universidad Autónoma de México. México.

Hernández Sampieri, Roberto, Carlos Fernández Collado y Pilar Baptista Lucio. 2006. Metodología de la Investigación. Editorial McGraw-Hill: México.

Instituto de Fomento y Asesoría Municipal (IFAM). 2010. Estudio sobre tendencias de desarrollo local. Inventario de organizaciones de base comunitaria 2010. IFAM. Costa Rica.

Instituto de Estadísticas y Censo (INEC). 2011. Resultados generales de $X$ censo de población y VI censo de vivienda y encuestas de propósitos múltiples a hogares. Instituto de Estadísticas y Censo. Costa Rica.

La Gaceta. 2012. Instituciones Descentralizadas. La Gaceta. Diario Oficial (193). Costa Rica.

Ministerio de Salud. 2005. Estudio de carga de enfermedad en Costa Rica. Dirección de Vigilancia de la Salud. Proyecto BID-MS. Costa Rica

Moya, Ligia. 2009. Introducción a la Estadística de la salud. Editorial Universidad de Costa Rica: Costa Rica.

Municipalidad de Osa. 2013. Programa Municipal de Gestión del Sector Pesquero Artesanal y Piangüero del Cantón de Osa. Costa Rica.

Organización de las Naciones Unidas (ONU). 2009. "Capítulo 17. Protección de los océanos y los mares de todo tipo incluidos los mares cerrados y semicerrados y de las zonas costeras y desarrollo de los recursos vivos". En Programa 21. ONU, EE. UU. 
Rojas, N., M. Alfaro, J. Solano, C. Araya y R. Villalobos. 2011. Cuenca Río Térraba. MINAET. Instituto Meteorológico Nacional. PNUD: Costa Rica

Reyes, V., M. Miranda, C. Monge y F. Salas. 2004. Valoración económica del ecosistema Humedal Nacional Térraba-Sierpe y Propuesta de mecanismos para su sostenibilidad. Costa Rica.

Sierra Segura, Claudine, Édgar Castillo Cruz y Stanley Arguedas Mora. 2007. Plan de Manejo del Humedal Nacional Térraba-Sierpe. Diagnósticos biofísicos, social, económico, productivo y análisis institucional. ACOSA, Nature Conservancy, ELAP, UCl: Costa Rica.

Simard M., K. Zhang, V. Rivera Monroy, M. Ross, P. Ruiz, E. CastañedaMoya,

Robert R. Twilley y E. Rodríguez. 2006. Mapping Height and Biomass of Mangrove Forest in Everglades National Park with SRTM Elevation Data Marc. Centro de Investigaciones Ambientales. Universidad Internacional de Florida. EE. UU.

Solís, V., P. Madrigal, D. Barguil, P. Cambronero. 2009. Sones que se van al mar y estrategias de manejo para la sobrevivencia. Un ejemplo de como también en Centroamérica se trata de rescatar la identidad cultural de la pesca artesanal. Coopesolidar: Costa Rica. 